\title{
Hydrothermal Synthesis of Ultrasmall Pt Nanoparticles as Highly Active Electrocatalysts for Methanol Oxidation
}

\section{Wenhai Ji ${ }^{1}$, Weihong Qi ${ }^{1,2,3, *}$, Shasha Tang ${ }^{1}$, Hongcheng Peng ${ }^{1}$ and Siqi $\mathrm{Li}^{1}$}

1 School of Materials Science and Engineering, Central South University, Changsha 410083, China; E-Mails: jwh1988@csu.edu.cn(W.J.); tang0360@ntu.edu.sg(S.T.); 133111028@csu.edu.cn (H.P.); 9901090160@csu.edu.cn (S.L.)

2 Institute for Materials Microstructure, Central South University, Changsha 410083, China

3 Key Laboratory of Non-Ferrous Materials Science and Engineering, Ministry of Education, Changsha 410083, China

* Author to whom correspondence should be addressed; E-Mail: qiwh216@csu.edu.cn; Tel.: +86-731-88876692.

Academic Editors: Hermenegildo García and Sergio Navalón

Received: 31 October 2015 / Accepted: 25 November 2015 / Published: 8 December 2015

\begin{abstract}
Ultrasmall nanoparticles, with sizes in the 1-3 $\mathrm{nm}$ range, exhibit unique properties distinct from those of free molecules and larger-sized nanoparticles. Demonstrating that the hydrothermal method can serve as a facile method for the synthesis of platinum nanoparticles, we successfully synthesized ultrasmall Pt nanoparticles with an average size of $2.45 \mathrm{~nm}$, with the aid of poly(vinyl pyrrolidone) (PVP) as reducing agents and capping agents. Because of the size effect, these ultrasmall Pt nanoparticles exhibit a high activity toward the methanol oxidation reaction.
\end{abstract}

Keywords: hydrothermal method; ultrasmall nanoparticles; PVP; methanol oxidation

\section{Introduction}

Platinum has been widely used in many applications, especially for catalysis and fuel cell technology, due to its ability to facilitate both oxidation and reduction reactions [1-5]. Size and shape play an important role in determining properties and potential applications of nanomaterials. Thus, downsizing these nanomaterials can provide a great opportunity to achieve a high surface-to-volume ratio and thus enhance the metal utilization in noble metal-based catalysts or electrocatalysts. 
Ultrasmall nanoparticles (USNPs), with sizes in the 1-3 nm range, exhibit unique properties distinct from those of free molecules and larger-sized nanoparticles.

Pt USNPs have also been reported. By using alcohols as reductants in the presence of poly(vinyl pyrrolidone) (PVP) in a refluxing aqueous system, Pt USNPs were obtained [6]. The average size of Pt USNPs could be controlled from 1.9 to $3.3 \mathrm{~nm}$ by changing the alcohol or the concentrations of the reagents. Photochemical methodology also could be used as a very effective way to produce Pt USNPs [7]. It involved visible light as a reaction trigger and platinum acetylacetonate and thioglycolic acid as the only chemical reactants. The average diameter of platinum nanoparticles was $1.0 \pm 0.3 \mathrm{~nm}$. Li et al. reported the synthesis of monodisperse Pt USNPs stabilized with peptides in aqueous solution at room temperature [8]. The specifically selected peptide molecule, P7A, is able to bind to the surface of the Pt NPs and regulate the nucleation and growth rates, affording monodisperse Pt NPs with sizes in the 1.7-3.5 nm range. Pt USNPs have also been synthesized with hydrophobic ligands. Uniform 2-nm-sized Pt USNPs were obtained by the decomposition of platinum dibenzylideneacetone under mild conditions in the presence of n-octylsilane [9]. Lim et al. successfully synthesized Pt USNPs with an average size of $1.9 \mathrm{~nm}$ through the reaction of a Pt salt precursor with water, without the aid of any exotic reducing agents and organic capping molecules [10]. The in situ synthesis of Pt USNPs at room temperature using poly(4-vinyl phenol) ( $\mathrm{PVPh})$ as both the reducing as well as the stabilizing agent in aqueous alkaline solution has been reported [11]. Transmission electron microscopic analysis confirms the formation of ultra-small spherical Pt USNPs from 1.6 to $2.2 \mathrm{~nm}$ in diameter with a high degree of monodispersity depending on the ratio of PVPh to platinum salt concentrations used in a single reaction.

Specific organic capping agents or stabilizers have been exploited in order to restrict nanocrystal growth as well as to provide a barrier to agglomeration. Notably, PVP has received special attention due to its high chemical stability, nontoxicity, and excellent solubility in many polar solvents. Hydroxyl end groups of PVP can be applied as a general strategy for the kinetically controlled synthesis of nanoplates made of noble metals such as Ag, Pd, Au, and Pt [12,13]. PVP (see Scheme 1) has an affinity for long-chain alcohols owing to its hydroxyl end groups; it can serve as a reducing agent. As a major advantage over alcohols with short alkyl chains commonly used in chemical synthesis, the PVP can be used as both reductant and stabilizer.

The hydrothermal method has been used in the synthesis of semiconductor nanocrystals and nanocomposites for a long time, but its role remains largely unexplored in the synthesis of noble-metal colloids. Here we demonstrate that Pt USNPs can be synthesized through a hydrothermal method with PVP. To the best of our knowledge, the present work may be the first report on synthesizing Pt USNPs in aqueous solutions by means of a simple hydrothermal method.

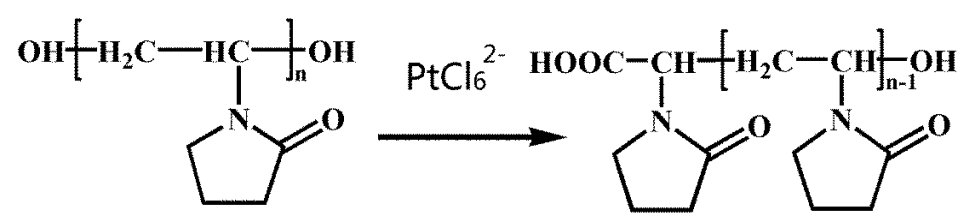

Scheme 1. Formulas of OH-terminated poly(vinyl pyrrolidone) (PVP) and the structural variation in the effect of $\mathrm{PtCl}_{6}^{2-}$ ions. 


\section{Results and Discussion}

We synthesized Pt USNPs by reacting $\mathrm{K}_{2} \mathrm{PtCl}_{6}$ with PVP in a closed autoclave in an oven at $160{ }^{\circ} \mathrm{C}$ for $8 \mathrm{~h}$. Transmission electron microscope (TEM) images in Figure 1a,b show that the Pt USNPs have an average size around $2.45 \mathrm{~nm}$. The high resolution TEM image of the crystal structure of the $\mathrm{Pt}$ USNPs in Figure 1c displays a well-defined crystal lattice. The inset image in Figure 1c shows that a d spacing between adjacent lattice planes of $0.224 \mathrm{~nm}$ corresponds to the $\{111\}$ planes, and $0.195 \mathrm{~nm}$ corresponds to the $\{200\}$ planes. TEM studies and a size histogram of Pt USNPs obtained by counting 200 particles (Figure 1d) show that these Pt USNPs typically have nearly spherical shapes, with a narrow size distribution. The formation of Pt USNPs synthesized in the hydrothermal method is due to the presence of PVP. The OH-terminated PVP can be applied as the reductant for the synthesis of nanoparticles made of other noble metals (such as $\mathrm{Ag}, \mathrm{Pd}, \mathrm{Au}$, and $\mathrm{Pt}$ ) [12-15]. Compared with alcohols (commonly used in the chemical synthesis), the PVP can be used as both a reductant and stabilizer. Scheme 1 shows the formula of $\mathrm{OH}$-terminated PVP and the structural changes effected by $\mathrm{PtCl}_{6}{ }^{2-}$ ions. The powder X-Ray diffraction (XRD) pattern of the product (Figure 2) was matched with that of bulk Pt (Fm-3m, a = 3.924, PDF No. 89-7382), confirming the formation of metallic Pt USNPs with a face-centered cubic structure.
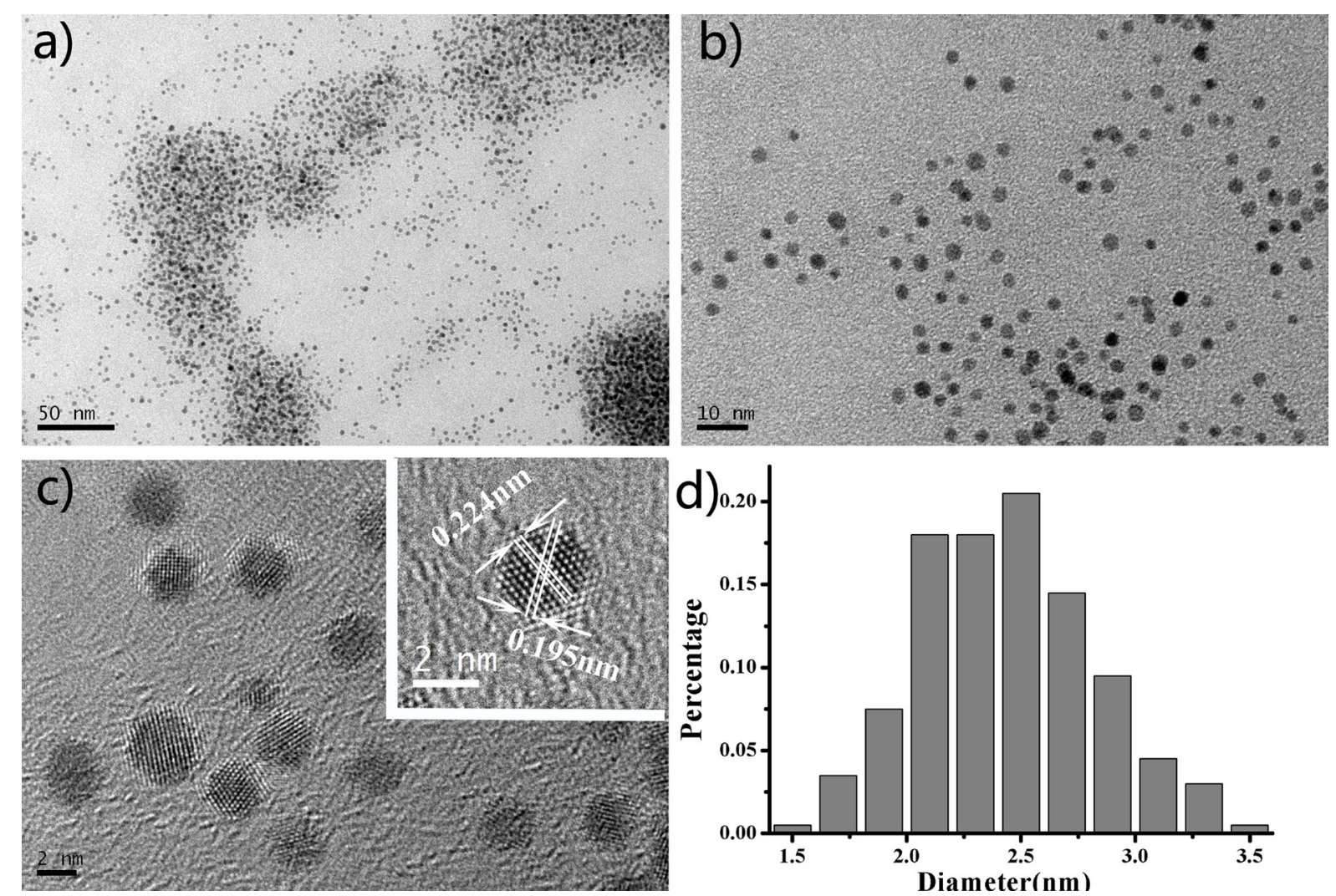

Figure 1. (a,b) Typical transmission electron microscope (TEM) images of ultrasmall Pt nanoparticles at different magnification and (c,d) high-resolution TEM image and the corresponding size distributions of the samples. 


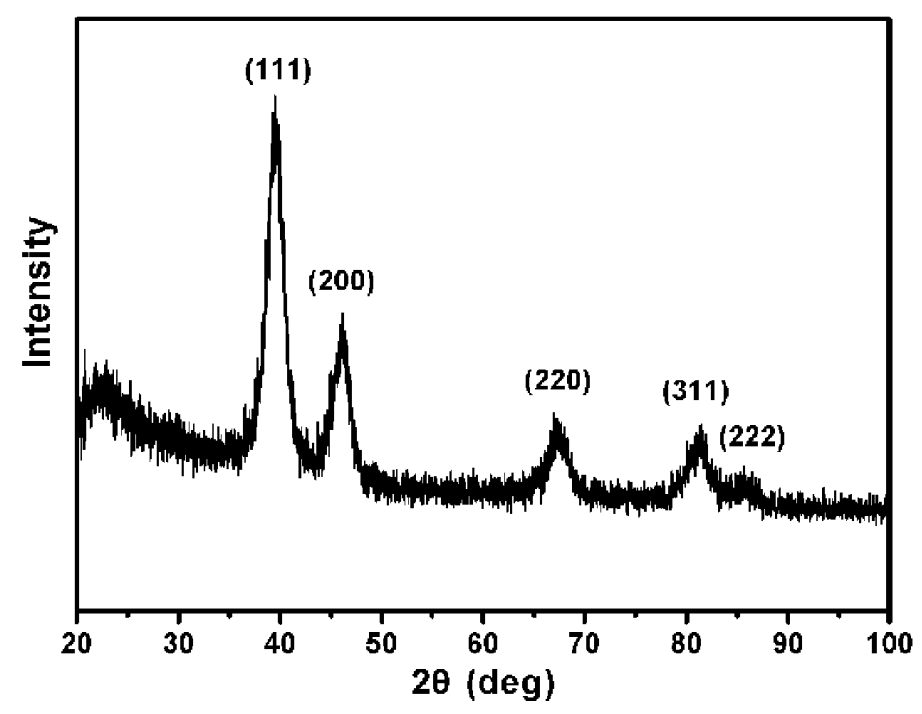

Figure 2. Powder X-Ray diffraction (XRD) pattern of ultrasmall Pt nanoparticles.

We tested the electrochemical behaviors of the prepared Pt USNPs. In order to minimize possible aggregation during the catalytic reaction and utilize the support effect, we used Vulcan XC-72 as the support. Vulcan XC-72 carbon, which is the most widely used support material for direct methanol fuel cell electrodes, is amorphous with a specific surface area of $212 \mathrm{~m}^{2} \cdot \mathrm{g}^{-1}$. A given weight of Vulcan $\mathrm{XC}-72$ was mixed with the as-prepared solution of Pt USNPs for an hour. The products were separated and dried in a vacuum oven. Figure 3 a shows that a number of Pt USNPs were distributed almost evenly over the entire surfaces of the Vulcan carbon powders. We measured cyclic voltammetry (CV) curves of Pt/Vulcan XC-72 catalysts in $\mathrm{N}_{2}$-saturated $0.5 \mathrm{M} \mathrm{H}_{2} \mathrm{SO}_{4}$ in comparison with the commercial $\mathrm{Pt} / \mathrm{C}$ catalyst (E-TEK, 30\% Pt on Vulcan XC-72 carbon support). CV curves of Pt/Vulcan XC-72 catalysts (Figure $3 \mathrm{~b}$ ) reveal a large peak between -0.24 and $0.08 \mathrm{~V}$ (vs. saturated calomel electrode (SCE)), which is associated with the hydrogen adsorption/desorption processes. The electrochemically active surface area (ECSA) can be calculated from the charge involved in the hydrogen adsorption/desorption processes using Equation (1) [16].

$$
E C S A=Q /[P t] \times 0.21 \mathrm{mC} \cdot \mathrm{cm}^{-2}
$$

where $Q(\mathrm{mC})$ and $[\mathrm{Pt}]$ are the charge for hydrogen adsorption/desorption and the loading of Pt on the electrode, respectively, while $0.21 \mathrm{mC} \cdot \mathrm{cm}^{-2}$ is the electrical charge associated with the monolayer adsorption of hydrogen on Pt [17]. The ECSA of the Pt/Vulcan XC-72 is as high as $46.2 \mathrm{~m} / \mathrm{gPt}_{\mathrm{Pt}}$, which is 1.52 times greater than that of the commercial $\mathrm{Pt} / \mathrm{C}$ catalyst $\left(30.3 \mathrm{~m}^{2} / \mathrm{gPt}_{\mathrm{Pt}}\right)$. It demonstrated $\mathrm{Pt} / \mathrm{Vulcan}$ $\mathrm{XC}-72$ catalysts had much higher ECSA.

The electrochemical performance of the Pt/Vulcan XC-72 composite (shown in Figure 3a) was tested for methanol oxidation, which is at the heart of direct methanol fuel cells (DMFC) application in the anodic half-cell reaction. For comparison, the commercial $\mathrm{Pt} / \mathrm{C}$ catalyst was also evaluated in the same condition. The electrocatalytic performances of the Pt-based electrocatalysts towards methanol oxidation were evaluated using $\mathrm{CV}$ and amperometric $i$ - $t$ in $0.5 \mathrm{M} \mathrm{H}_{2} \mathrm{SO}_{4}+0.5 \mathrm{M} \mathrm{CH}_{3} \mathrm{OH}$ solution. $\mathrm{CV}$ curves in Figure 3c show two prominent peaks in both positive and negative scans corresponding to the methanol oxidation for all tested catalysts, and it can be seen that the current from methanol oxidation becomes apparent as the potential rises to above $0.2 \mathrm{~V}$. In the forward scan, methanol 
oxidation produces an anodic peak at around $0.6 \mathrm{~V}$. In the reverse scan, an anodic peak appears at around $0.4 \mathrm{~V}$. This anodic peak in the reverse scan can be attributed to the removal of the incompletely oxidized carbonaceous species formed in the forward scan. These features of CV curves are in agreement with the reports for the $\mathrm{Pt} / \mathrm{C}$ catalyst. As shown in Figure 3c, the peak current of methanol oxidation on Pt/Vulcan XC-72 is $373 \mathrm{~mA} \cdot \mathrm{mg}^{-1} \mathrm{Pt}$, which is much higher than that of the commercial $\mathrm{Pt} / \mathrm{C}$ catalyst $\left(148.3 \mathrm{~mA} \cdot \mathrm{mg}^{-1} \mathrm{Pt}\right)$. The ratio of the forward oxidation current peak $\left(I_{\mathrm{f}}\right)$ to the reverse current peak $\left(I_{\mathrm{b}}\right), I_{\mathrm{f}} / I_{\mathrm{b}}$, is an index of the catalyst tolerance to the poisoning species. A higher ratio indicates more effective removal of the poisoning species on the catalyst surface. The $I_{\mathrm{f}} / I_{\mathrm{b}}$ ratio of $\mathrm{Pt} /$ Vulcan XC-72 is 2.27, higher than that of the commercial Pt/C (1.93), showing better catalyst tolerance of the Pt/Vulcan XC-72 catalyst.
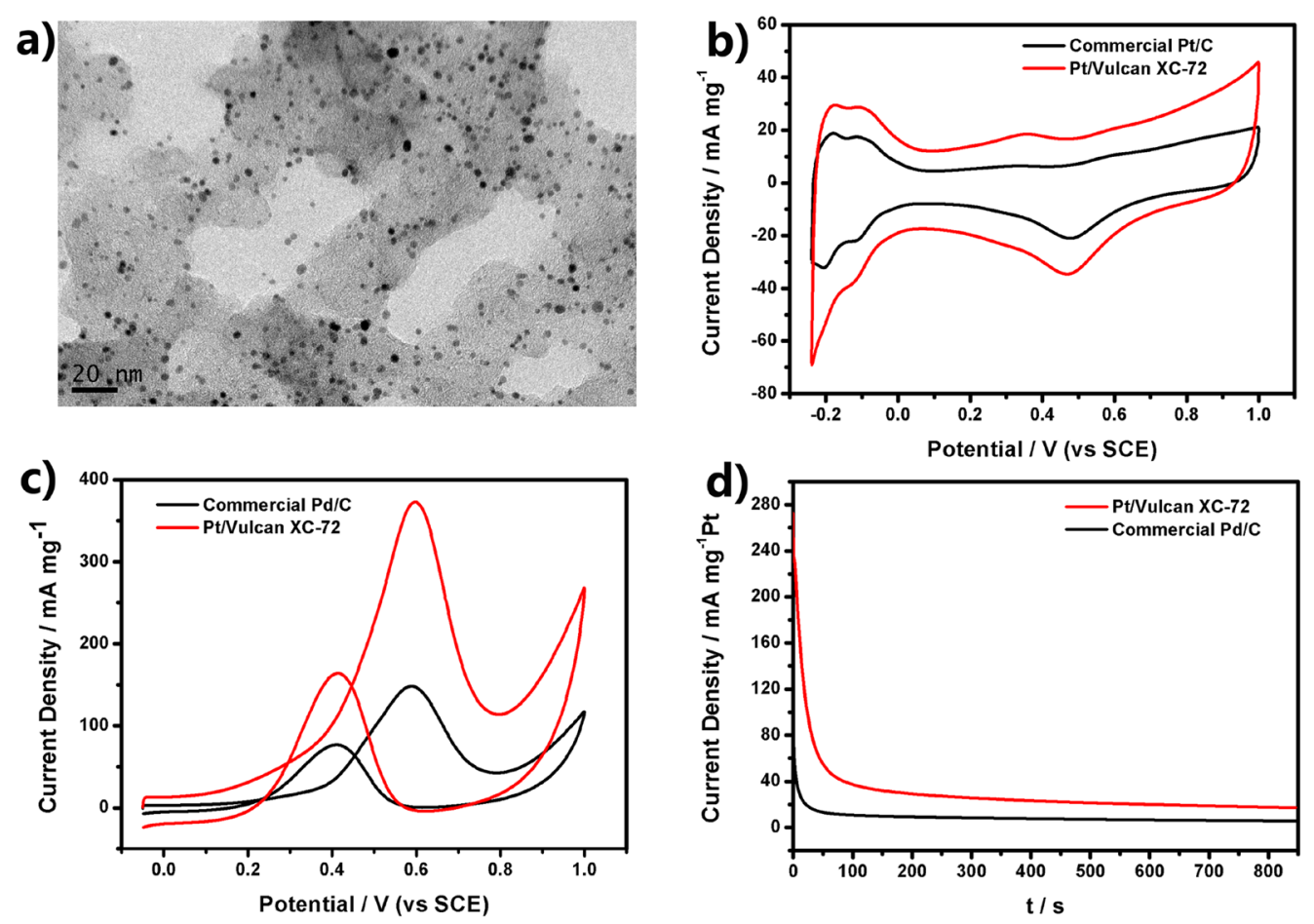

Figure 3. (a) TEM images of Pt ultrasmall nanoparticles (USNPs) supported on Vulcan XC-72 carbon; (b) Cyclic voltammetry (CV) curves of Pt electrocatalysts in $\mathrm{N}_{2}$-saturated $0.5 \mathrm{M} \mathrm{H}_{2} \mathrm{SO}_{4}$ with a scan rate of $50 \mathrm{mV} \cdot \mathrm{s}^{-1}$; (c) $\mathrm{CV}$ curves at a scan rate of $50 \mathrm{mV} \cdot \mathrm{s}^{-1}$ with different Pt electrocatalysts in $0.5 \mathrm{M} \mathrm{H}_{2} \mathrm{SO}_{4}+0.5 \mathrm{M} \mathrm{CH}_{3} \mathrm{OH}$; (d) Amperometric $i$ - $t$ curves of Pt electrocatalysts in $0.5 \mathrm{M} \mathrm{H}_{2} \mathrm{SO}_{4}+0.5 \mathrm{M} \mathrm{CH}_{3} \mathrm{OH}$ solution at $0.6 \mathrm{~V}$ (vs. saturated calomel electrode (SCE)).

To further compare the electrochemical long-term performance between Pt/Vulcan XC-72 and commercial $\mathrm{Pt} / \mathrm{C}$, a chronoamperometry test was conducted in the solution of $0.5 \mathrm{M} \mathrm{H}_{2} \mathrm{SO}_{4}$ and $0.5 \mathrm{M}$ $\mathrm{CH}_{3} \mathrm{OH}$ for $850 \mathrm{~s}$ at a fixed potential of $0.6 \mathrm{~V}$ (vs. SCE), as shown in Figure $3 \mathrm{~d}$. The initial high current density was attributed to the double-layer charging and the numerous available active sites of Pt atoms. The currents of all the catalysts decreased rapidly within first few seconds due to the formation of CO-like intermediates such as $\mathrm{CO}$ and $\mathrm{CHO}$, etc. [18-21], which generated during the oxidation of methanol and adsorbed on the surface of active Pt atoms to prevent methanol's further 
oxidation on these Pt atoms. After that, the currents kept on decreasing and gradually maintained a steady state. The current density of Pt/Vulcan XC-72 was higher than that of commercial Pt/C during the whole testing time. The current density of Pt/Vulcan XC-72 is $17.2 \mathrm{~mA} \cdot \mathrm{mg}^{-1}$ at $850 \mathrm{~s}$, which is much higher than that of commercial $\mathrm{Pt} / \mathrm{C}\left(5.6 \mathrm{~mA} \cdot \mathrm{mg}^{-1}\right.$ at $\left.850 \mathrm{~s}\right)$. The higher current density directly reflected the higher resistivity of $\mathrm{Pt} / \mathrm{Vulcan} \mathrm{XC}-72$ to the poisoning of CO-like intermediates.

\section{Experimental Section}

\subsection{Chemicals and Materials}

Potassium hexachloroplatinate (IV) $\left(\mathrm{K}_{2} \mathrm{PtCl}_{6}, \mathrm{AR}\right)$ and methanol were purchased from Shanghai Chemical Reagent Company (Shanghai, China). Poly(vinyl pyrrolidone) (PVP, $M_{\mathrm{w}}=55,000$ ) was received from Aldrich. Vulcan XC-72 was purchased from Cabot (Boston, MA, USA) and 5\% Nafion was purchased from Sigma (Shanghai, China). All chemicals were used as received without further purification. High-purity deionized water $(>18.4 \mathrm{M} \Omega \cdot \mathrm{cm})$ was produced using Millipore A10 Milli-Q (Darmstadt, Germany).

\subsection{Synthesis of Pt USNPS}

A typical synthesis process can be concisely described as follows. $\mathrm{K}_{2} \mathrm{PtCl}_{6}(38.8 \mathrm{mg})$ and PVP (44.4 $\left.\mathrm{mg}, M_{\mathrm{w}}=55,000\right)$ were added into water $(10 \mathrm{~mL})$, and the solution was stirred for $10 \mathrm{~min}$. Then the homogeneous orange-yellow solution was transferred to a $15 \mathrm{~mL}$ Teflon-lined stainless steel autoclave. The container was then sealed in a stainless steel bomb. The whole system was heated and maintained in an oven at $160{ }^{\circ} \mathrm{C}$ under autogenous pressure for $8 \mathrm{~h}$. After the reaction finished, the container was cooled under room temperature conditions naturally. Finally, a solution of Pt particles with dark gray color was obtained.

\subsection{Synthesis of Pt USNPS/Vulcan XC-72}

A given weight of Vulcan XC-72 was mixed with the as-prepared solution of Pt USNPs for an hour. The products were separated via centrifugation $(12,000 \mathrm{rpm}, 10 \mathrm{~min})$ and further purified twice by deionized water and then dried in a vacuum oven at $80^{\circ} \mathrm{C}$ for $8 \mathrm{~h}$.

\subsection{Characterization}

The transmission electron microscopy (TEM) images were obtained on JEOL JEM-2100F instruments (Tokyo, Japan) operating at an accelerating voltage of $200 \mathrm{kV}$. For the preparation of samples for electron microscopy analyses, aliquots taken from the as-prepared Pt USNPs were dropped directly onto carbon-coated copper grids placed on a filter paper and then dried at room temperature in air. The grids were placed in a sack and steeped in alcohol for a half hour. After that, the grids were taken out and dried at room temperature in air. The structures of as-prepared products were characterized by $\mathrm{X}$-Ray diffraction (XRD) (Rigaku D/Max $2550 \mathrm{X}, \mathrm{Cu} \mathrm{K} \alpha$ radiation, $\lambda=0.154178 \mathrm{~nm}$, Tokyo, Japan). The composition of Pt/Vulcan XC-72 was determined by energy dispersive X-Ray spectroscopy (EDS, Quanta FEG 250, 20 kV, FEI Company, Hillsboro, OR, USA). 


\subsection{Electrocatalytic Activity Evaluation}

Electrochemical characterizations were carried out using with CHI660D and a three-electrode configuration with a saturated calomel electrode (SCE) and a platinum foil as the reference and counter electrode, respectively. The catalyst ink, which was prepared from the mixture of catalyst ( $2 \mathrm{mg}$ ), ethanol $(1 \mathrm{~mL})$, and Nafion solution $(5 \%, 50 \mu \mathrm{L})$, was spread onto a glass carbon (GC) electrode (4 mm diameter). For all electrodes, a metal loading of $23.8 \mu \mathrm{g} \cdot \mathrm{cm}^{-2}$ was used. Commercial $\mathrm{Pt} / \mathrm{C}$ catalyst (30 wt \%) was also tested for comparison. Cyclic voltammetry (CV) measurements were performed in $0.5 \mathrm{M} \mathrm{H}_{2} \mathrm{SO}_{4}$ solutions under a flow of $\mathrm{N}_{2}$ at a sweep rate of $50 \mathrm{mV} \cdot \mathrm{s}^{-1}$. The amperometric $i$ - $t$ curves were obtained in $0.5 \mathrm{M} \mathrm{H}_{2} \mathrm{SO}_{4}+0.5 \mathrm{M} \mathrm{CH}_{3} \mathrm{OH}$.

\section{Conclusions}

In summary, a novel method has been successfully developed to synthesize Pt USNPs with an average size of $2.45 \mathrm{~nm}$. These Pt USNPs supported on Vulcan XC-72 exhibited an enhanced activity for methanol oxidation compared to the commercial $\mathrm{Pt} / \mathrm{C}$ catalyst, thus providing great potential as a promising electrocatalyst for high performance DMFCs. Our results suggest that the PVP-assisted hydrothermal method is a simple and environmentally benign route for the synthesis of $\mathrm{Pt}$ nanoparticles for catalysis and other applications. Significantly, our results also provide a new insight into the role played by the hydrothermal method in the synthesis of noble metal nanoparticles.

\section{Acknowledgments}

This work was supported by National Nature Science Foundation of China (No. 21373273), Hunan Provincial Natural Science Foundation of China (No. 13JJ1002), Fundamental Research Funds for the Central Universities of Central South University (No. 2014zzts168) and Shenghua Scholar Program of Central South University.

\section{Author Contributions}

Wenhai Ji performed the experimental and sample preparation, general data mining and analysis, and prepared the manuscript. Shasha Tang, Hongcheng Peng and Siqi Li performed the electrochemical characterizations and data analysis. This project was proposed by Weihong Qi, who also supervised the research process, discussed the experimental results, revised the manuscript and approved the final version.

\section{Conflicts of Interest}

The authors declare no conflict of interest.

\section{References}

1. Mourdikoudis, S.; Chirea, M.; Altantzis, T.; Pastoriza-Santos, I.; Pérez-Juste, J.; Silva, F.; Bals S. Liz-Marzán, L.M. Dimethylformamide-mediated synthesis of water-soluble platinum nanodendrites for ethanol oxidation electrocatalysis. Nanoscale 2013, 5, 4776-4784. 
2. Peng, Z.; Kisielowski, C.; Bell, A.T. Surfactant-free preparation of supported cubic platinum nanoparticles. Chem. Commun. 2012, 48, 1854-1856.

3. Tian, N.; Zhou, Z.Y.; Sun, S.G.; Ding, Y.; Wang, Z.L. Synthesis of tetrahexahedral platinum nanocrystals with high-index facets and high electro-oxidation activity. Science 2007, 316, 732-735.

4. Chen, A.; Holt-Hindle, P. Platinum-based nanostructured materials: Synthesis, properties, and applications. Chem. Rev. 2010, 110, 3767-3804.

5. Matoh, L.; Škofic, I.K.; Čeh, M.; Bukovec, N. A novel method for preparation of a platinum catalyst at low temperatures. J. Mater. Chem. A 2013, 1, 1065-1069.

6. Teranishi, T.; Hosoe, M.; Tanaka, T.; Miyake, M. Size control of monodispersed Pt nanoparticles and their 2D organization by electrophoretic deposition. J. Phys. Chem. B 1999, 103, 3818-3827.

7. Giuffrida, S.; Ventimiglia, G.; Callari, F.L.; Sortino, S. Straightforward Light-Driven Synthesis of Ultrasmall, Water-Soluble Monolayer-Protected Platinum Nanoparticles. Eur. J. Inorg. Chem. 2006, 20, 4022-4025.

8. Li, Y.; Whyburn, G.P.; Huang, Y. Specific peptide regulated synthesis of ultrasmall platinum nanocrystals. J. Am. Chem. Soc. 2009, 131, 15998-15999.

9. Pelzer, K.; Hävecker, M.; Boualleg, M.; Candy, J.P.; Basset, J.M. Stabilization of 200-Atom Platinum Nanoparticles by Organosilane Fragments. Angew. Chem. 2011, 123, 5276-5279.

10. Lim, G.H.; Yu, T.; Koh, T.; Lee, J.H.; Jeong, U.; Lim, B. Reduction by water for eco-friendly, capping agent-free synthesis of ultrasmall platinum nanocrystals. Chem. Phys. Lett. 2014, 595, 77-82.

11. Maji, T.; Banerjee, S.; Biswas, M.; Mandal, T.K. In situ synthesis of ultra-small platinum nanoparticles using a water soluble polyphenolic polymer with high catalytic activity. $R S C A d v$. 2014, 4, 51745-51753.

12. Xiong, Y.; Washio, I.; Chen, J.; Cai, H.; Li, Z.Y.; Xia, Y. Poly(vinyl pyrrolidone): A dual functional reductant and stabilizer for the facile synthesis of noble metal nanoplates in aqueous solutions. Langmuir 2006, 22, 8563-8570.

13. Washio, I.; Xiong, Y.; Yin, Y.; Xia, Y. Reduction by the end groups of poly(vinyl pyrrolidone): A new and versatile route to the kinetically controlled synthesis of Ag triangular nanoplates. Adv. Mater. 2006, 18, 1745-1749.

14. Ji, W.; Qi, W.; Tang, S.; Huang, B.; Wang, M.; Li, Y.; Jia, Y.; Pang, Y. Synthesis of Marks-Decahedral Pd Nanoparticles in Aqueous Solutions. Part. Part. Syst. Charact. 2014, 31, $851-856$.

15. Ji, W.; Qi, W.; Li, X.; Zhao, S.; Tang, S.; Peng, H.; Li, S. Investigation of disclinations in Marks decahedral Pd nanoparticles by aberration-corrected HRTEM. Mater. Lett. 2015, 152, 283-286.

16. Pozio, A.; de Francesco, M.; Cemmi, A.; Cardellini, F.; Giorgi, L. Comparison of high surface $\mathrm{Pt} / \mathrm{C}$ catalysts by cyclic voltammetry. J. Power Sources 2002, 105, 13-19.

17. Maillard, F.; Martin, M.; Gloaguen, F.; Léger, J.M. Oxygen electroreduction on carbon-supported platinum catalysts. Particle-size effect on the tolerance to methanol competition. Electrochim. Acta 2002, 47, 3431-3440.

18. Lee, S.H.; Kakati, N.; Jee, S.H.; Maiti, J.; Yoon, Y.S. Hydrothermal synthesis of PtRu nanoparticles supported on graphene sheets for methanol oxidation in direct methanol fuel cell. Mater. Lett. 2011, 65, 3281-3284. 
19. Prabhuram, J.; Zhao, T.S.; Tang, Z.K.; Chen, R.; Liang, Z.X. Multiwalled carbon nanotube supported PtRu for the anode of direct methanol fuel cells. J. Phys. Chem. B 2006, 110, 5245-5252.

20. Zhang, Y.; Gu, Y.E.; Lin, S.; Wei, J.; Wang, Z.; Wang, C.; Du, Y.; Ye, W. One-step synthesis of PtPdAu ternary alloy nanoparticles on graphene with superior methanol electrooxidation activity. Electrochim. Acta 2011, 56, 8746-8751.

21. Liu, F.; Lee, J.Y.; Zhou, W.J. Segmented Pt/Ru, Pt/Ni, and Pt/RuNi nanorods as model bifunctional catalysts for methanol oxidation. Small 2006, 2, 121-128.

(C) 2015 by the authors; licensee MDPI, Basel, Switzerland. This article is an open access article distributed under the terms and conditions of the Creative Commons Attribution license (http://creativecommons.org/licenses/by/4.0/). 